\title{
Particle Simulation of Charging Characteristic of a Micro-sized Dust Particle Immersed into Low Temperature Plasma
}

\author{
Z. Qian \\ School of Petroleum Engineering \\ Changzhou University \\ China
}

\author{
H. Wang \\ School of Petroleum Engineering \\ Changzhou University \\ China
}

\begin{abstract}
The charging characteristic of micro-sized particulates immersed into low temperature argon plasma was studied via particle simulation employing Particle-in-Cell (PIC) and Monte-Carlo (MC) hybrid method. The net charge of a dust particle was determined by counting the number of electrons and ions reached the particle surface. The electric potential of particle surface was evaluated by the capacitor model. Meanwhile, the profiles of charged particle number densities and self-consistent electric field were obtained. Both electron and ion number densities dramatically varied near the particle surface within the sheath. The simulation was verified by comparing the computational results with the data from existing simulation and theoretical analysis. The comparison showed that the numerical model overestimating the charge number for micro-sized particles. The research indicates that the charge of a particle is proportional to its radius and the electron temperature. However, the relationship is not strictly linear.
\end{abstract}

Keywords- dust particle; charging; low temperature plasma; particle simulation

\section{INTRODUCTION}

Charging of dust particles in low temperature plasma is a concern of dusty plasma. The charging characteristic of dust particles are interested in air purification, aerospace engineering, plasma enhanced chemical vapor deposition, powder technology, and etc. The topic has been studied both experimentally [1 3] and theoretically [4 6]. The experimental work is focused on measuring the charge of particulate under different discharging conditions, back pressures, and dust concentration. The theoretical study of dusty plasma has developed some analytical solutions to evaluate the charges of dust particles. However, there is still hard to accurately predicting the charges of a fine particulate.

Meanwhile, lots of numerical efforts [7 10] have been applied in simulating gas discharging and plasma transportation. Particle simulation based on Monte-Carlo and Particle-in-Cell hybrid method [9 12] is inherently inexpensive and effective has become a main trend for studying dusty plasma dynamics. Here the PIC-MC hybrid method is employed to simulate the charging process and predict the charges of fine dust particles immersed into low temperature plasma. The effort of this paper is referable for further study.

\section{NUMERICAL MODEL}

In this simulation, the dust particles are treated as stationary, spherical shaped, and isolated. Moreover, dust motion is not included, dust temperature is fixed, and dust-dust interaction is excluded. A $1 \mathrm{D} 3 \mathrm{~V}$ model is adopted due to the symmetrical property of a spherical coordinate system, which drastically reduced the computation. The simulation is mainly composed of four parts: 1) initialization and establishing boundary condition; 2) particle motion; 3) collision computation; 4) electric field solving and particle acceleration.

\section{A. Boundary Conditions}

The boundary conditions adopted in the simulation are as follows

$$
\begin{array}{cc}
\varphi=Q /\left(4 \pi \varepsilon_{0} R\right) & r=R \\
\partial n / \partial r=0 & r=R \\
\varphi=0 & r=L \\
n=n_{i 0}=n_{e 0} & r=L
\end{array}
$$

Where $\varphi$ is the electric potential, $Q$ is the net charge of the target particle surface, $R$ is the radius of the particle, $\varepsilon_{0}$ is the dielectric constant, $r$ is the spherical coordinate, $L$ is the length of domain, $n$ is the number density of pseudo-particles, and $n_{i 0} 、 n_{e 0}$ are background number densities of ion and electron, respectively.

Initially, both ions and electrons are uniformly distributed in the computational domain and under thermal equilibrium condition. The treatment on the boundary is that: 1) once an ion (or electron) reaches the surface of the target particle, i.e. $r$ $\leq R$, its charge is transferred to particle and uniformly distributed on the surface. Then, it is removed; 2) if an ion moves out the zone, it will be removed, too. The parameters used in simulation are given in Table 1.

TABLE I. PARAMETERS FOR COMPUTATION

\begin{tabular}{|c|c|}
\hline item & value \\
\hline background gas & $\mathrm{Ar}$ \\
\hline pressure & $133 \mathrm{~Pa}$ \\
\hline electron number density & $10^{15} / \mathrm{m}^{3}$ \\
\hline ion number density & $10^{15} / \mathrm{m}^{3}$ \\
\hline electron temperature & $23200 \mathrm{~K}$ \\
\hline ion temperature & $300 \mathrm{~K}$ \\
\hline
\end{tabular}




\section{B. Movements of Electrons and Ions}

The simulation particles are advanced by the Verlet Scheme. In the light of that the mass of an ion are much larger than that of an electron, the time steps for ions and electrons are $5.0 \times 10^{-12} \mathrm{~s}$ and $1.0 \times 10^{-8} \mathrm{~s}$, respectively. In order to facilitate the simulation, the computation is terminated at $t=3.0 \times 10^{-5} \mathrm{~s}$. (the estimated charging time is $\sim 10^{-5} \mathrm{~s}[5]$ )

\section{Treatment of Collision Events}

The collisions between charged pseudo-particles, i.e. ions and electrons, and background neutrals are modeled. The ionization, excitation, and elastic scattering events are also included. The free path based rule is adopted to determine whether a collision is happed or not [9]. Also the null collision scheme is employed to judge the type of collision events [10]. Then the post collision velocities of simulation particles are calculated [11].

\section{Solving the Electric Field}

The Ruyten method [12] is employed for charge assignment in a spherical coordinate system. After accumulating charges to nodes, the charge densities of the nodes are obtained. Then, the electric field could be calculated by solving the Poisson Equation with TDMA scheme [11]. Thus, the charge of the particulate is determined by the capacitor model. Furthermore, the same assignment principle is applied to evaluate the electric force of charged particles in the cells.

\section{RESULTS AND DISCUSSION}

The convergence history of the calculation for a particulate with a radius of $5 \mu \mathrm{m}$ is shown in Fig. 1. It is confirmed that the charging time is about $10^{-5} \mathrm{~s}$. The net charge of particulate surface is about $-27800 e$ (elementary charge). And the profile of space potential is obtained. As shown in Fig. 2, the surface potential is about $-8.0 \mathrm{~V}$. And the potential dramatically increases with the increment of distance from the center of the particle $r$. The voltage varies from $-8.0 \mathrm{~V}$ to $-1.0 \mathrm{~V}$ within 30 $\mu \mathrm{m}$ ( Debye length). However, once $r$ is greater than $50 \mu \mathrm{m}$, the potential slightly varies with the distance and tends to be 0 $\mathrm{V}$ within $100 \mu \mathrm{m}$.

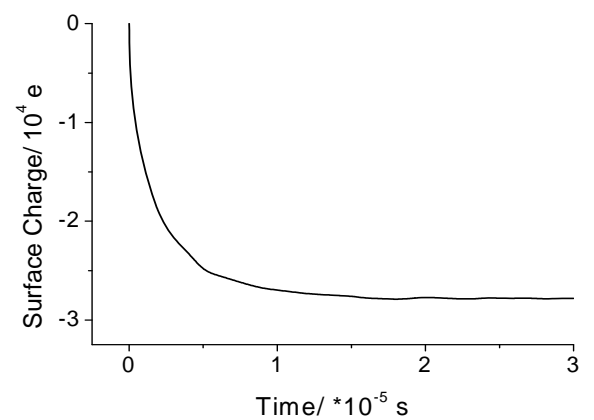

FIGURE I. SURFACE CHARGE VERSUS TIME FOR A PARTICULATE WITH THE RADIUS OF $5 \mathrm{MM}$.

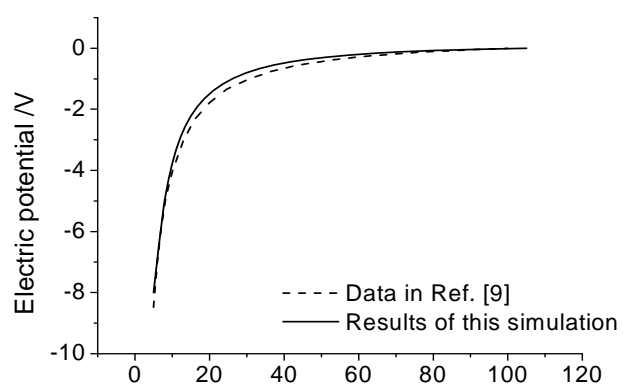

Distance from the center of the particulate $/ 10^{-6} \mathrm{~m}$

FIGURE II. PROFILES OF ELECTRIC POTENTIAL ( $\mathrm{R}=5 \mathrm{MM})$.

In order to validate the numerical model, a comparison of results among different models is listed in Table 2 . The computational results are consistent with the predictions of Ref. [9] and [4]. However, the difference between this simulation and model of Ref. [6] are observed. It should be noted that both electron temperature and ion temperature are included in Ref. [6].

TABLE II. COMPARISON OF SURFACE POTENTIAL OF A DUST PARTICLE WITH A RADIUS OF $5 \mathrm{MM}$

\begin{tabular}{|c|c|}
\hline data source & surface potential (V) \\
\hline this simulation & -8.0 \\
\hline Ref. [9] & -8.6 \\
\hline Ref. [4] & -9.4 \\
\hline Ref. [6] & -4.2 \\
\hline
\end{tabular}

The plasma number density around the dust particle is shown in Fig. 3. Both the electron and the ion densities rapidly rise up to the background plasma parameters with the increment of distance. It is noticed that $n_{i}$ is large than $n_{e}$ throughout the near field, though the difference between them becomes smaller as the distance is large enough. Furthermore, the variation of the $n_{i}$ can be divided into two stages, i.e. if $r<$ $30 \mu \mathrm{m}, n_{i}$ sharply increases from $\sim 1 \times 10^{14} \mathrm{~m}^{-3}$ to $9 \times 10^{14} \mathrm{~m}^{-3}$ within $25 \mu \mathrm{m}$. Whereas, if $r>30 \mu \mathrm{m}, n_{i}$ slightly goes up. On the other hand, $n_{e}$ rises relatively slowly and evenly. When the distance is as large as $\sim 110 \mu \mathrm{m}$, the two densities tends to be equal, which indicates that the sheath thickness around the particulate wall is about $105 \mu \mathrm{m}$ ( 3 Debye length).

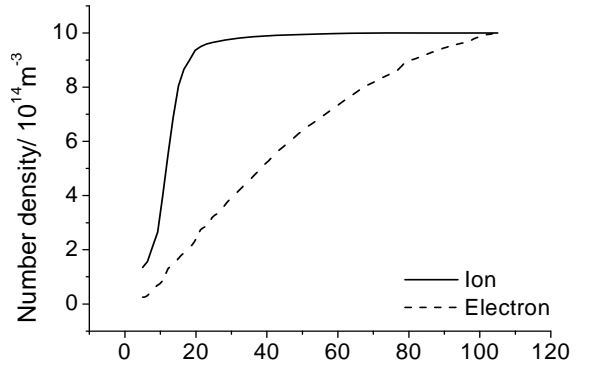

Distance from the center of the particulate $/ 10^{-6} \mathrm{~m}$

FIGURE III. PROFILES OF PARTICLE NUMBER DENSITIES ( $\mathrm{R}=5 \mathrm{MM})$.

Figure 4 reveals that the net charge of a dust particle are proportion to its radius, which is caused by the capacitor model for solving electric filed. And the dust particle is negatively charged because the electrons move faster than the ions. Some 
electrons reach the dust surface in the early stage and establish the electric field, which affects the motion of the other ions and electrons. Fig. 4 also indicates that the charge of a micro-sized particle is not big in the low temperature plasma, if the radius of the particle is less than $2.5 \mu \mathrm{m}, Q$ is less then $-10,000 e$. which means traditional electrostatic precipitator is difficult to remove such fine particles.

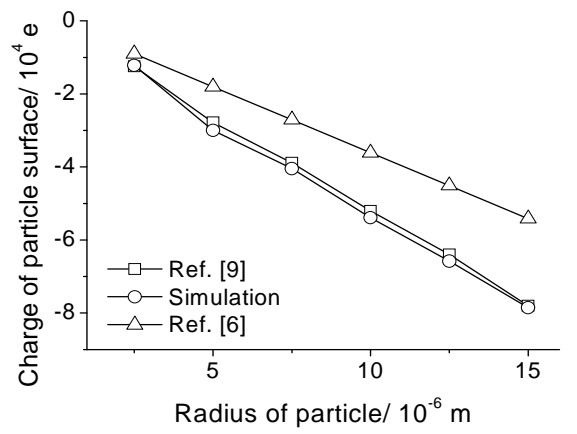

FIGURE IV. CHARGE QUANTITY VERSUS PARTICLE RADIUS.

According to the existing theoretical analysis, the electron temperature is a major factor affecting dust charging while the temperature of ions is a minor factor. It is approximately recognized that $Q$ is proportion to $T_{e}$. Here, the effect of the electron temperature is studied and shown in Fig. 5. It confirms that a higher electron temperature leads to a higher charge quantity for a dust particulate. And the ratio of charges with different electron temperature is about 1.3 1.4, which is less than that of electron temperatures $(3 / 2=1.5)$.

Future work should include dust motion, dust-dust interaction, and dust temperature. Also a cluster of dusts should be tracked simultaneously. However, such a complicated model is a heavy load for current simulation.

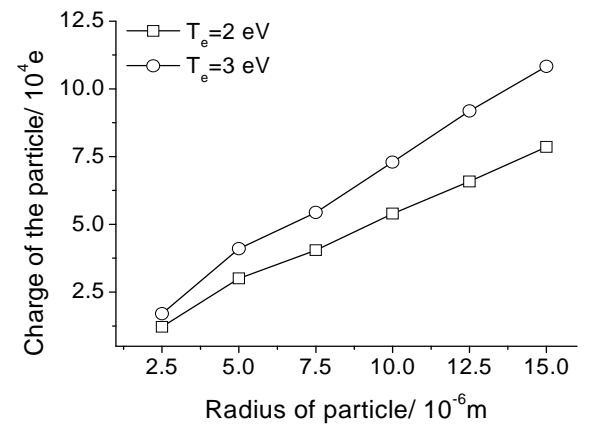

FIGURE V. CHARGES OF DUST PARTICLES WITH DIFFERENT ELECTRON TEMPERATURE.

\section{CONCLUSIONS}

In all, the following conclusions are reached: 1) the particle surface has the minimum electric potential and the space voltage close to the particle rapidly goes to $0 \mathrm{~V}$ within several Debye lengths; 2) both electron and ion number densities around dust surface increase with the increment of distance from particle center. Ion density is evidently larger than electron density throughout the computational zone, the former has a sharp increase in the vicinity of particle surface. Finally, both of them achieve the background plasma parameter; and 3) the charge quantity is proportional to the particle size. As for a particulate with a radius of $5 \mu \mathrm{m}$, the charge quantity is about $27800 e$ corresponding to the surface potential of approximately $-8.0 \mathrm{~V}$.

\section{ACKNOWLEDGMENTS}

The work of this paper is supported by Doctoral Fund of Ministry of Education of China (Grant No. 20103120120003) and Ministry of Education of Jiangsu (Grant No. 12KJB470001).

\section{REFERENCES}

[1] Smith, M.A., Goodrich, J., Rahman, H.U., et al., Measurement of grain charge in dusty plasma Coulomb crystals. IEEE Transaction on Plasma Science, 29(2), pp. 216-220, 2001.

[2] Tomme, E.B., Law, D.A., Annaratone, B.M., et al., Parabolic plasma sheath potentials and their implications for the charge on levitated dust particles. Phys. Rev. Lett., 85(12), pp. 2518-2521, 2000.

[3] Liu, B., Goree, J., Fortov, E., et al., Dusty plasma diagnostics methods for charge, electron temperature, and ion density. Physics of Plasmas, 17(5), pp. 053701, 2010.

[4] Lieberman, M.A., \& Lichtenberg, A.J., Principles of plasma discharges and materials processing, John Wiley \& Sons, Inc., New Jersey, 2005.

[5] Shukla, P.K., \& Mamun, A.A., Introduction to Dusty Plasma Physics. Plasma Phys. Control. Fusion, 44(3), 2003.

[6] Matsoukas, T., \& Russell, M., Particle charging in low pressure plasmas. J. Appl. Phys., 77(9), pp. 4285-4292, 1995.

[7] Dorranian, D., Shahbaz Tahmasebi, F., Sari, A.H., et al., Calculation of electrical potential and dust particle charging a double dusty plasma device. J. Fusion Energy, 30(1), pp. 16-20, 2011.

[8] Pandey, B.P., Vladimirov, S.V., \& Samarian, A.A., Charge on the dust in the plasma. PHYSICAL REVIEW E, 83(1), pp. 016401, 2011.

[9] Bartos, P., Blazek, S., Jelinek, P., et al., Hybrid computer simulations: electrical charging of dust particles in low-temperature plasma. Eur. Phys. J. D, 54(2), pp. 319-323, 2009.

[10] Donko, Z., Particle simulation methods for studies of low-pressure plasma sources. Plasma Sources Sci. Technol., 20(2), pp. 024001, 2011.

[11] Birdsall, C.K., Particle-in-Cell charged-particle simulation, plus Monte Carlo collisions with neutral atoms, PIC-MCC. IEEE Transactions on Plasma Science, 19(2), pp. 65-85, 1991.

[12] Ruyten, W.M., Density-conserving shape factors for particle simulations in cylindrical and spherical coordinates. Journal of Computation Physics, 105(2), pp. 224-232, 1993 